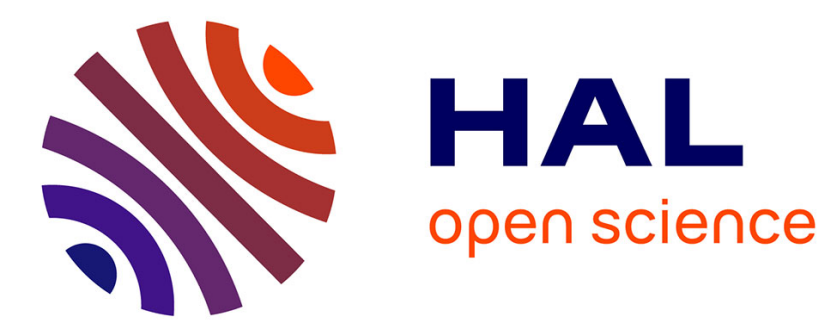

\title{
Some Basics of Unilateral Dynamics
}

Jean Jacques Moreau

\section{- To cite this version:}

Jean Jacques Moreau. Some Basics of Unilateral Dynamics. IUTAM Symposium on Unilateral Multibody Contacts, Aug 1998, Munich, Germany. hal-01793543

\section{HAL Id: hal-01793543 \\ https://hal.science/hal-01793543}

Submitted on 16 May 2018

HAL is a multi-disciplinary open access archive for the deposit and dissemination of scientific research documents, whether they are published or not. The documents may come from teaching and research institutions in France or abroad, or from public or private research centers.
L'archive ouverte pluridisciplinaire HAL, est destinée au dépôt et à la diffusion de documents scientifiques de niveau recherche, publiés ou non, émanant des établissements d'enseignement et de recherche français ou étrangers, des laboratoires publics ou privés. 
Private draft of a preliminary version of: (F. Pfeiffer and Ch. Glocker, eds.) Unilateral Multibody

Contacts, Kluwer Academic Publishers, Dordrecht, 1999, pp. 1-14

\title{
SOME BASICS OF UNILATERAL DYNAMICS
}

\author{
J. J. MOREAU \\ Laboratoire de Mécanique et Génie Civil \\ Case 048, Université Montpellier II, 34095 Montpellier Cedex, \\ France.
}

\section{Introduction}

In this Proceedings volume various situations are met in which the dynamical motion of collections of bodies subject to unilateral constraints of non-interpenetrability has to be calculated.

After the pioneering work of P. Cundall [3], motivated by geomechanical problems, the most popular numerical techniques used for this purpose depend on the approximate replacement of the unilateral constraints by repulsion laws which enter into action as soon as a pair of bodies come sufficiently close to each other. Also, some additional effects governed by nonsmooth laws, such as dry friction and possible collisions may be approximated through analogous regularization procedures. The dynamics of the approximate system is then governed by differential equations with sufficient regularity to be handled through standard numerical techniques.

The drawback is that the need of precision calls for the use of stiff approximate laws. Hence the time-stepping schemes applied have to resort to very small step-length and possibly also have to enforce numerical stability by introducing artificial damping or artificially increasing inertia. In some applications, the effect of such alterations of the mechanical data may blur the picture. For this reason, a number of authors [11][14][2][15][1] have preferred to base the computation on the strict handling of unilaterality and nonsmoothness. It was not before a paper of E. Delassus [4] that some attention was paid to the problems raised by the discussion of contact persistence when several unilateral constraints are present. As Delassus' arguments today seem difficult to read, the same questions were investigated again in 
[8][9] with the help of Convex Analysis and Complementarity theory, which currently remain the favorite tools in the domain.

In the author's experience, the rather subtle concurrence of inequality conditions and of evolutions governed by differential equations has generated frequent misunderstandings. The present paper is meant to clear up certain points without exhibiting too cumbersome technicalities.

\section{The differential handling of inequality conditions}

\subsection{TWO LOCAL STATEMENTS}

Let $\varphi$ denote a real function defined on the interval $[0, T[$ with $\varphi(0)=0$ and the right-derivative $\varphi^{\prime+}(0)$ assumed to exist. The definition of this derivative readily entails the following.

- If $\varphi \geq 0$ on a neighbourhood of 0 in $\left[0, T\left[\right.\right.$, then $\varphi^{\prime+}(0) \geq 0$.

- If $\varphi^{\prime+}(0)>0$, there exists an interval $] 0, \epsilon[$ on which $\varphi>0$.

Clearly, the respective converses of these two implications are false.

Let $t \in[0, T$ [ be the time and let $\varphi(t)$ express the normal gap between two bodies which lie in contact at instant 0 . The above shows that the prediction of contact to persist or to break cannot be reduced to the evaluation of derivatives at $t=0$. Invoking derivatives of order higher than one, if they exist, does not evade the occurence of some non conclusive cases.

In these local statements, we have considered the operation of derivating a real function as a point operation. In the usual theory of Differential Equations, the derivative of a function, considered globally over a whole interval, is rather viewed as 'what allows one to retrieve the function through integration', a standpoint emphasized below.

\subsection{PRIMARY EXAMPLE OF A DIFFERENTIAL INCLUSION}

Let us investigate a moving point in $\mathbf{R}^{n}$, i.e. a mapping $q:\left[0, T\left[\rightarrow \mathbf{R}^{n}\right.\right.$. We want $q(t)$ to comply for every $t$ with some inequality requirement

$$
f(t, q(t)) \leq 0
$$

where $f:\left[0, T\left[\times \mathbf{R}^{n} \rightarrow \mathbf{R}\right.\right.$ denotes a $\mathcal{C}^{1}$ real function. It is assumed that, for every $t$ in $\left[0, T\left[\right.\right.$ and every $x$ in $\mathbf{R}^{n}$, the gradient $\nabla f(t, x):=\left(\partial f / \partial x^{1}, \ldots\right.$, $\partial f\left(\partial x^{n}\right)$ is not identically zero. Put

$$
\Gamma(t, x):=\left\{\begin{array}{l}
\left\{v \in \mathbf{R}^{n} \mid \partial f / \partial t+v \cdot \nabla f(t, x) \leq 0\right\} \quad \text { if } \quad f(t, x) \geq 0 \\
\mathbf{R}^{n} \text { otherwise. }
\end{array}\right.
$$

Suppose $q$ is locally absolutely continuous on the interval [0,T[. Equivalently, the derivative $d q / d t$ exists almost everywhere and equals a locally 
Lebesgue-integrable function $u:\left[0, T\left[\rightarrow \mathbf{R}^{n}\right.\right.$ such that, for every $t \in[0, T[$, one has $q(t)=q(0)+\int_{0}^{t} u(s) d s$.

THE INTEGRATION LEMMA [11]. Assume that inclusion

$$
\frac{d q}{d t} \in \Gamma(t, q(t))
$$

holds for almost every $t$ in $[0, T[$ and that inequality (1) is verified for $t=0$. Then the same inequality is verified for every $t \in[0, T[$.

Proof. Put $\varphi(t)=f(t, q(t))$, a locally absolutely continuous real function, since $f$ is $\mathcal{C}^{1}$. Through the chain rule, its derivative is expressed almost everywhere as $\varphi^{\prime}=\partial f / \partial t+u . \nabla f$. Imagine the existence of $\tau$ in $[0, T[$ such that $\varphi(\tau)>0$ and look for contradiction.

Since $\varphi(0) \leq 0$, continuity imposes on $\varphi$ to take the value 0 on a nonempty subset of $[0, \tau[$. Let $\sigma$ denote the l.u.b. of this subset; by continuity $\varphi(\sigma)=0$, while $\varphi$ is positive on the interval $] \sigma, \tau]$. As $u=d q / d t$ verifies $(2), \varphi^{\prime}$ should be $\leq 0$ almost everywhere on this interval, hence $\varphi(\tau)=\int_{\sigma}^{\tau} \varphi^{\prime}(t) d t \leq 0$, which is a contradiction.

A condition of the form (2), imposed on a function $q:\left[0, T\left[\rightarrow \mathbf{R}^{n}\right.\right.$, is called a differential inclusion.

By imagining some selection rules for the multifunction $(t, x) \rightarrow \Gamma(t, x)$, one may construct differential equations whose solutions a fortiori verify (2). Here is a simple example.

\subsection{SWEEPING}

Let $\Gamma$ be defined as above. For every $(t, x)$ in $\left[0, T\left[\times \mathbf{R}^{n}\right.\right.$, denote by $\gamma(t, x)$ the element of minimal norm in $\Gamma(t, x)$. If $f(t, x)<0$, this simply equals the zero of $\mathbf{R}^{n}$. If $f(t, x) \geq 0$, the set $\Gamma(t, x)$ is a half-space which contains the origin or not, depending on the sign of $\partial f / \partial t$. If the latter is $\leq 0, \gamma(t, x)$ again equals the zero of $\mathbf{R}^{n}$. Otherwise, the zero of $\mathbf{R}^{n}$ lies outside of the half-space and $\gamma(t, x)=-\left((\partial f / \partial t) /\|\nabla f\|^{2}\right) \nabla f$.

The motion of a point $q$ verifying the differential equation

$$
\frac{d q}{d t}=\gamma(t, q(t))
$$

and initially $f(0, q(0)) \leq 0$ may be described as follows. Owing to the Integration Lemma, $q$ belongs for every $t$ to the moving region $\Phi(t):=\{x \in$ $\left.\mathbf{R}^{n} \mid f(t, x) \leq 0\right\}$. As long as it lies in the interior, the point stays at rest. It is only when the boundary of $\Phi(t)$, i.e. the hypersurface with equation $f=0$, moves inward and reaches $q$ that the point takes on a velocity in 
inward normal direction, so as to go on belonging to $\Phi(t)$. The magnitude of this velocity vector equals the 'normal speed' of the hypersurface.

Here is another characterization of this motion. If, at time $t$, a point $x$ lies in the hypersurface $f=0$, the vector $\nabla f(t, x)$ (we have assumed it nonzero) is known to be normal to this hypersurface and directed outward of the region $\Phi(t)$. The half-line emanating from the origin, generated by $\nabla f(t, x)$, is said to constitute the normal cone to $\Phi(t)$ at point $x$; notation: $N_{\Phi(t)}(x)$ (the definition of a normal cone for less regular sets than $\Phi$ is a vast subject; some other cases will be met in the sequel). For $x$ in the interior of $\Phi(t)$, it proves consistent to define $N_{\Phi(t)}(x)$ as reduced to the zero of $\mathbf{R}^{n}$, while the cone shall be defined as empty if $x \notin \Phi(t)$. By discussing the various cases occuring in the calculation of $\gamma(t, x)$, one sees that every solution $q$ to the differential equation (3) verifies, for almost every $t$, the differential inclusion of the Sweeping Process [7][13]

$$
-\frac{d q}{d t} \in N_{\Phi(t)}(q(t)) .
$$

Unexpectedly, the converse is true, i.e. the inclusion (4), in spite of its right-hand side not reducing to a single element, actually is equivalent to the differential equation (3).

In fact let $q$, locally absolutely continuous of $\left[0, T\right.$ [into $\mathbf{R}^{n}$, be a solution to (4). For almost every $t$, the derivative $d q / d t$ exists, so the right-hand side is nonempty and, consequently, $q(t) \in \Phi(t)$. The same is true for every $t$, by continuity. For $t$ such that $q(t)$ lies in the interior of $\Phi(t),(4)$ implies $d q / d t=0$, which makes that (3) is also satisfied. Otherwise, suppose that $q(t)$ belongs to the boundary, i.e. the function $\tau \rightarrow f(\tau, q(\tau))$ vanishes at $\tau=t$. Then the right-derivative $\partial f / \partial t+q^{\prime+}(t) . \nabla f(t, q(t))$, if it exists, is $\leq 0$ while, symmetrically, the left-derivative is $\geq 0$. Therefore the bilateral derivative $q^{\prime}(t)$, which exists with the possible exception of a Lebesgue-negligible subset of $\left[0, T\left[\right.\right.$, satisfies $\partial f / \partial t+q^{\prime}(t) . \nabla f(t, q(t))=0$, i.e. it belongs to the boundary of the half-space $\Gamma(t, q(t))$. Furthermore, (4) entails that this vector is directed as the inward normal to the half-space, a property which elementarily characterizes it as equal to $\gamma(t, q(t))$.

\section{Duality}

\subsection{PAIRED LINEAR SPACES}

Let $X$ and $Y$ denote two real linear spaces. The concept of a bilinear form, say $x \in X, y \in Y \mapsto\langle x, y\rangle \in \mathbf{R}$, placing these spaces in duality has long been implicit in Mechanics: if $X$ is a space of velocities and $Y$ a space of forces, such a bilinear form expresses the power associated with the pair $x, y$. Equivalently, the elements of $X$ may be infinitesimal displacements, 
that is the products of velocities by a certain formal time-increment, in which case the bilinear form expresses an infinitesimal work.

This mathematical structure takes its full interest with the infinitedimensional spaces of Functional Analysis and the connection of duality with the definition of topologies on these spaces. In this paper, we shall restrict ourselves to finite-dimensional spaces, so that the definition of topologies on $X$ and $Y$ is standard and unambiguous, securing the continuity of every linear function. For brevity, we suppose that the duality is separating, i.e. every linear form on $X$ can uniquely be represented as $x \mapsto\langle x, y\rangle$, with $y \in Y$, and symmetrically with $X$ and $Y$ exchanged. In other words, by referring to the bilinear form $\langle.,$.$\rangle , one may identify each of the two spaces$ $X$ and $Y$ with the dual of the other.

As soon as some bases are chosen in the respective spaces, the bilinear form may be calculated from the components of the two vectors as $\langle x, y\rangle=$ $b_{i j} x^{i} y^{j}$. A simplification occurs if the two bases are suitably paired: with every base in one of the spaces, another one is uniquely associated in the other space, called the conjugate base, such that $b_{i j}=\delta_{i j}$, i.e. the expression of $\langle x, y\rangle$ reduces to the standard scalar product $x^{i} y^{i}$ of $\mathbf{R}^{n}$.

\subsection{INNER PRODUCT AND SELF-DUALITY}

As a special case, $X$ and $Y$ may consist of the same space, say $E$, so that the bilinear form becomes an inner product, more commonly denoted with a simple dot. In this case, it makes sense to assume the symmetry $x . y=y \cdot x$. If, in addition, $x . x>0$ for every nonzero element $x$ (an assumption which is found to secure that the corresponding duality of $E$ with itself is separating) the linear space $E$ is said Euclidean.

Such is the space of the vectors of the three-dimensional space of elementary Geometry and Physics, denoted in the sequel by $\mathbf{E}_{3}$.

Many usual operations performed in $\mathbf{R}^{n}$ rest on the standard Euclidean structure of this $n$-dimensional linear space. So is, for instance, the construction of a pseudo-inverse for a noninvertible linear map, a tool sometimes useful in the handling of mechanical constraints. Actually, the mechanical problems in view intrinsically pertain to some linear spaces, the representation of which in terms of $\mathbf{R}^{n}$ results from the choice of a base. In common practice, this choice is based on calculation convenience, so no mechanical significance should be attributed to such Euclidean operations. 


\section{Parametrization of a Multibody System}

\subsection{CONFIGURATIONS AND VELOCITIES}

Let the possible configurations of a mechanical system be parametrized, at least locally, through generalized coordinates, say $q^{1}, \ldots, q^{n}$. The choice of this parametrization is governed by convenience and any differentiable change of parameters is mechanically admissible. In other words, the set $\mathcal{Q}$ of the possible configurations of the system may be viewed as a Differential Manifold. Such a close connection exists between Analytical Dynamics and Differential Geometry that, in spite of computation finally dealing only with elements of $\mathbf{R}^{n}$, we think it a good precaution to keep some basic geometrical concepts in mind. For instance, this may prevent from paying undue attention to calculation artefacts devoid of mechanical significance.

Define a motion of the system by making $q^{1}, \ldots, q^{n}$ depend on the time $t$ in a differentiable way. For every instant, say $t_{1}$, the values of the $n$ derivatives $q^{i \prime}\left(t_{1}\right)$ are commonly called the generalized velocity components of the system. If the differential geometric standpoint is adopted, these $n$ real numbers properly are the components of an element of some abstract $n$-dimensional linear space, called the tangent space at the considered point $q$ of $\mathcal{Q}$, that we shall denote by $\mathcal{Q}_{q}^{\prime}$. If a differentiable change of parameters is performed, the time-derivatives at $t_{1}$ of the new parameters, for the same motion as before, are connected with the time-derivatives of the former ones through linear formulas, the coefficients of which constitute the Jacobian matrix of the change of variable. From the differential geometric standpoint, this linear transform merely reflect a change of base in the tangent space: the time derivatives of the $n$ new parameters are the new components of the same element of $\mathcal{Q}_{q}^{\prime}$ as before, intrinsically called the system velocity.

When the considered motion is smoooth enough for the second derivatives $q^{i \prime \prime}\left(t_{1}\right)$ to exist, it is common in Computation literature to refer to these quantities as the 'acceleration components', but one should recall that, in case of a change of parameters, they do not remain the components of a definite element of $\mathcal{Q}_{q}^{\prime}$. In fact, the first derivatives $q^{i \prime}(t)$ equal the components of the velocity, an element of $\mathcal{Q}_{q(t)}^{\prime}$, but the latter does not consist of the same linear space when $t$ varies, so one cannot rely on the traditional concept of the derivative of a vector function.

\subsection{CONTACT KINEMATICS}

For the sake of reducing the number $n$, the parametrization $\left(q^{1}, \ldots, q^{n}\right)$ may be constructed with account of possible permanent, frictionless linkages imposed on the members of the system. After that, the constraints of noninterpenetrability are additionally considered. The consequent geometric 
restriction on the system positions is assumed expressed by a finite set of inequalities

$$
f_{\alpha}(t, q) \leq 0, \quad \alpha \in\{1, \ldots, \kappa\},
$$

where $f_{1}, \ldots, f_{\kappa}$ are given functions. Through the presence of $t$ as an argument of $f_{\alpha}$, provision is made for the inequality to describe the confinement of a part of the system by some external boundary with prescribed motion.

Suppose that inequality $f_{\alpha} \leq 0$ expresses the local non-interpenetration of some pair of members of the system, say $\mathcal{B}$ and $\mathcal{B}^{\prime}$, so that equality $f_{\alpha}=0$ correspond to these bodies touching each other at some point of space denoted by $M_{\alpha}$. This we shall assume to be an isolated contact point, but other contacts, associated with different values of $\alpha$, may also be in effect between the same bodies at the same instant. For every imagined motion $t \mapsto q(t)$ bringing the system through the considered contacting position for some value of $t$, the velocity vectors $\mathcal{V}_{\alpha}$ and $\mathcal{V}_{\alpha}^{\prime}$, relative to the chosen reference frame, of the respective particles of $\mathcal{B}$ and $\mathcal{B}^{\prime}$ passing at point $M_{\alpha}$ let themselves be expressed as affine functions of the system velocity $u \in \mathcal{Q}_{q}^{\prime}$. The same is thus true for the relative velocity $\mathcal{U}_{\alpha}=\mathcal{V}_{\alpha}-\mathcal{V}_{\alpha}^{\prime}$ of $\mathcal{B}$ with respect to $\mathcal{B}^{\prime}$ at this point, say

$$
\mathcal{U}_{\alpha}=G_{\alpha} u+\mathcal{W}_{\alpha},
$$

where $G_{\alpha}: \mathcal{Q}_{q}^{\prime} \rightarrow \mathbf{E}_{3}$ denotes a linear mapping, depending on $t$ and $q$. No attention is paid at this stage to the imagined motion preserving contact or not. The term $\mathcal{W}_{\alpha} \in \mathbf{E}_{3}$, a known function of $t$ and $q$, vanishes in the familiar case of a time-independent parametrization.

Similar formula holds if inequality $f_{\alpha} \leq 0$ expresses the confinement of a part $\mathcal{B}$ of the system by some external boundary with prescribed motion. Assume that equality $f_{\alpha}=0$ corresponds to contact taking place at some point, here again denoted by $M_{\alpha}$. The local velocity, at this point, of the body $\mathcal{B}$ with respect to the boundary has the same form as $\mathcal{U}_{\alpha}$ in (6), where $\mathcal{W}_{\alpha}$ now reflects the known velocity of the boundary (for a time-independent parametrization, $\mathcal{W}_{\alpha}$ equals the negative of this velocity vector).

At the contact point $M_{\alpha}$, we assume that a common tangent plane to the respective surfaces of the concerned bodies has been defined (this does not require of both surfaces to be smooth; for instance, contact may take place between a smooth body and the extremity of a spike of the other). Let $\mathbf{n}^{\alpha}$ denote the unit vector to this plane, directed toward $\mathcal{B}$. In computation, as well as in existential studies, it proves useful that the definition of the above elements would be conventionally extended to a neighbourhood of the concerned value of $(t, q)$ in $\mathbf{R} \times \mathcal{Q}$. This allows one to express as a function of $(t, q)$ the normal gap, say $g_{\alpha}(t, q)$, between $\mathcal{B}$ and $\mathcal{B}^{\prime}$, counted as negative in the case of overlap. Classically, the derivative of the function $t \mapsto g_{\alpha}(t, q(t))$ 
is found equal to $\mathcal{U}_{\alpha} \cdot \mathbf{n}^{\alpha}$, the normal component of the relative velocity of the contacting bodies at point $M_{\alpha}$.

Sometimes, in Computation literature, the second derivative of the same function is improperly referred to as the 'normal relative acceleration'. Actually, since the material particles involved in the definition of $\mathcal{U}_{\alpha}$ are not the same from an instant to the other, this second derivative has in general nothing to do with the relative acceleration vector.

\subsection{THE COTANGENT SPACE}

Let a real function $F$ be defined on the manifold $\mathcal{Q}$ and let $\hat{F}: \mathbf{R}^{n} \rightarrow \mathbf{R}$ its expression in the parametrization $\left(q^{1}, \ldots, q^{n}\right)$. If $\hat{F}$ is differentiable at a point of $\mathbf{R}^{n}$, the function $F$ is said differentiable at the corresponding point $q$ of $\mathcal{Q}$ and the partial derivatives $\partial \hat{F} / \partial q^{i}$ are declared to constitute the components of its gradient, denoted by $\nabla F$. The latter is an element of some linear space $\mathcal{Q}_{q}^{\prime *}$, the cotangent space to $\mathcal{Q}$ at point $q$. If, similarly to that was done in the preceding, a differentiable change of parameters is performed, the function $F$ assumes a new representation, and the new partial derivatives may be calculated from the former ones through linear formulas. These partial derivatives make the components of the same object $\nabla F$ as before relatively to a new base of $\mathcal{Q}_{q}^{\prime *}$.

The relationship between $\mathcal{Q}_{q}^{\prime}$ and $\mathcal{Q}_{q}^{\prime *}$ is made clear by considering the same moving point as in Subsec. 4.1, passing through the considered configuration at time $t_{1}$. The derivative of the real function $t \mapsto F(q(t))$ is expressed through the chain rule as $q^{i \prime}\left(t_{1}\right) \partial \hat{F} / \partial q^{i}$. That this derivative should not depend on the parametrization in use results in the following statements:

- a bilinear form, denoted by $\langle.,$.$\rangle , places the two spaces in duality,$

- the elements $q^{i \prime}\left(t_{1}\right)$ and $\partial \hat{F} / \partial q^{i}$ of $\mathbf{R}^{n}$ respectively make the components of $q^{\prime}\left(t_{1}\right)$ in $\mathcal{Q}_{q}^{\prime}$ and of $\nabla F$ in $\mathcal{Q}_{q}^{\prime *}$ relatively to a pair of bases which are conjugate with regard to this bilinear form,

- the derivative of $t \mapsto F(q(t))$ at $t_{1}$ equals $\left\langle q^{\prime}\left(t_{1}\right), \nabla F\right\rangle$.

\subsection{NORMALITY}

Assume that the configuration $q$ of the system at time $t$ satisfies $f_{\alpha}(t, q)=0$, i.e. the contact with label $\alpha$ is in effect. For this value of $t$, the points of $\mathcal{Q}$ satisfying the same equality make a hypersurface $\mathcal{S}_{\alpha}$. If the element $\nabla f_{\alpha}(t, q)$ of $\mathcal{Q}_{q}^{\prime *}$ is nonzero, it may be said normal to this hypersurface in the following sense: for every differentiable chain of points, say $\hat{q}: \mathbf{R} \rightarrow \mathcal{S}_{\alpha}$ such that $\hat{q}(0)=q$, this element is orthogonal, in the sense of the duality form $\langle.,$.$\rangle , to the tangent vector \hat{q}^{\prime}(0) \in \mathcal{Q}_{q}^{\prime}$. This immediately results from the expression given above to the chaine rule. Furthermore, $\nabla f_{\alpha}(t, q)$ is also 
able to meet the same need as the gradient does in the traditional Euclidean setting, that of distinguishing between the two sides of the hypersurface. In fact, if a differentiable chain of points $\tau \mapsto \hat{q}(\tau) \in \mathcal{Q}$, with $\hat{q}(0)=q$, satisfies $\left\langle\hat{q}^{\prime}(0), \nabla f_{\alpha}(t, q)\right\rangle>0$, then the point $q$ separates an arc $\tau<0$ which lies in the region $f_{\alpha}<0$ from an arc $\tau>0$ which lies in the region $f_{\alpha}>0$.

Let $\Phi(t)$ denote the feasible region at time $t$, i.e. the set of the positions complying with the non-interpenetration conditions (5). Put

$$
J(t, q):=\left\{\alpha \in\{1, \ldots, \kappa\} \mid f_{\alpha}(t, q) \geq 0\right\} .
$$

If $q \in \Phi(t)$, this is the set of the values of $\alpha$ such that the condition $f_{\alpha}(t, q) \leq 0$ holds as equality. The (outward) normal cone $N_{\Phi(t)}$ is defined as the convex cone generated in $\mathcal{Q}_{q}^{* *}$ by the elements $\nabla f_{\alpha}(t, q)$ with $\alpha \in J(t, q)$. This generated cone reduces to the zero of $\mathcal{Q}_{q}^{* *}$ if $J(t, q)$ is empty, i.e. if $q$ lies in the interior of $\Phi(t)$.

\subsection{CONTACT FORCES}

The technique of Analytical Dynamics requires that every collection of forces, say $\mathbf{e}_{1}, \mathbf{e}_{2}, \ldots \in \mathbf{E}_{3}$, applied to particles of the system in a given configuration $q$ be represented, with regard to the parametrization $q^{1}, \ldots, q^{n}$ in use, by $n$ real numbers $c_{1}, \ldots, c_{n}$, called the covariant components (or 'generalized components') of this collection of forces. The definition of this element of $\mathbf{R}^{n}$ rests, similarly to that was done in the preceding Subsec., on the consideration at fixed $t$, of a differentiable chain of configurations $\tau \mapsto \hat{q}(\tau) \in \mathcal{Q}$, with $\hat{q}(0)=q$ : this is traditionally called a virtual displacement at time $t$. Correspondingly, the material particle to which the forces are applied have positions $P_{1}(\tau), P_{2}(\tau), \ldots$, relative to the chosen reference frame, which depends differentiably on the variable $\tau$. The derivatives $P_{1}^{\prime}(0), P_{2}^{\prime}(0), \ldots \in \mathbf{E}_{3}$, called the virtual velocities of these particles are used to calculate $P_{1}^{\prime}(0) \cdot \mathbf{e}_{1}+P_{2}^{\prime}(0) \cdot \mathbf{e}_{2}+\ldots$ (Euclidean scalar products in $\left.\mathbf{E}_{3}\right)$, called the virtual power. This is required to equal $\hat{q}^{i \prime}(0) . c_{i}$, whatever the differentiable chain $\hat{q}$ is. Consequently, the $c_{i}$ are the components of an element of $\mathcal{Q}_{q}^{\prime *}$ independent of the parametrization in use.

There is no conceptual difficulty in extending this definition of the covariant components to more general 'efforts' than simple forces, for example to the concentrated torques commonly invoked in the phenomenological description of the resistance to rolling: the power of such a torque is defined through the spin vector of the concerned body.

What precedes applies in particular to the situation of Subsec.4.2. Two members $\mathcal{B}$ and $\mathcal{B}^{\prime}$ of the system being in contact at point $M_{\alpha}$, the contact force $\mathcal{R}^{\alpha}$ exerted by $\mathcal{B}^{\prime}$ upon $\mathcal{B}$ and its counterpart $-\mathcal{R}^{\alpha}$ exerted by $\mathcal{B}$ upon $\mathcal{B}^{\prime}$ together have a virtual power equal to the scalar product of $\mathcal{R}^{\alpha}$ by the 
relative virtual velocity of the contacting bodies at point $M_{\alpha}$. Expressing the latter from the derivative $\hat{q}^{\prime}(0)$ requires the same calculation as for Equ. (6), except that, the virtual displacement taking place at fixed $t$, the term $\mathcal{W}_{\alpha}$ is absent. Therefore, in the differential geometric setting, the pair of contact forces is represented by the element $r^{\alpha}$ of $\mathcal{Q}_{q}^{\prime *}$ such that $\left\langle\hat{q}^{\prime}(0), r^{\alpha}\right\rangle=G_{\alpha} \hat{q}^{\prime}(0) \cdot \mathcal{R}^{\alpha}$, whatever is $\hat{q}^{\prime}(0)$ in $\mathcal{Q}_{q}^{\prime}$, i.e.

$$
r^{\alpha}=G_{\alpha}^{*} \mathcal{R}^{\alpha},
$$

where the linear mapping $G_{\alpha}^{*}: \mathbf{E}_{3} \rightarrow \mathcal{Q}_{q}^{\prime *}$ equals the transpose of $G_{\alpha}$ with regard to the duality $\left\langle\mathcal{Q}_{q}^{\prime}, \mathcal{Q}_{q}^{\prime *}\right\rangle$ and to the self-duality of $\mathbf{E}_{3}$.

Similar formula is found to hold if equality $f_{\alpha}=0$ characterizes the contact of a part $\mathcal{B}$ of the system with some external boundary with prescribed motion. Then $r^{\alpha}$ in (8) makes the covariant representation of the force $\mathcal{R}^{\alpha}$ alone, acting on $\mathcal{B}$. Its counterpart exerted by $\mathcal{B}$ upon the boundary is not a force experienced by the system.

\section{Dynamics}

\subsection{LAGRANGE EQUATIONS}

The system Dynamics is governed by

$$
A(t, q) \frac{d u}{d t}=F(t, q, u)+\sum_{\alpha} r^{\alpha},
$$

where $A$ denotes the inertia matrix. The expression $F$ comprises standard terms of the Lagrange equations and the covariant components of some applied forces, supposed given as functions of the time, the position of the system and its velocity. The elements $r^{\alpha}, \alpha \in\{1,2, \ldots, \kappa\}$, are made of the covariant components of the contact forces as expressed in (8).

Equ. (9) must be read as an equality of elements of $\mathbf{R}^{n}$ (in the differential geometric formalism, as explained before, the symbol $d u / d t$ would have no meaning). The components of the velocity function $t \mapsto u$ do not necessarily equal the derivatives of the components of the configuration function $t \mapsto q$. For instance, when dealing with 3-dimensional rigid bodies, it is usual to attach to each of them a frame of principal axes of inertia emanating from its center of mass. Then one may choose to enter, as members of the $\mathbf{R}^{n}$-valued function $u$, the components relative to these axes of the spin vector of the rigid body, instead of the time-derivatives of some directional parameters. This amounts to use in the tangent space $\mathcal{Q}_{q}^{\prime}$ a base different from the one induced by the parametrization of $\mathcal{Q}$, with the considerable advantage of generating a contribution in the matrix $A$ which is diagonal and constant with regard to $t$ and $q$. Retrieving from the spin components the evolution of 
the directional parameters of the rigid body is only the matter of integrating some standard kinematical formulas.

To the above equation, the geometric conditions (5) of non-interpenetration are to be adjoined and also some phenomenological information concerning each possible contact, called a contact law, for which we assume the form

$$
\operatorname{law}_{\alpha}\left(t, q, \mathcal{U}_{\alpha}, \mathcal{R}^{\alpha}\right)=\text { true. }
$$

The local relative velocity $\mathcal{U}_{\alpha}$ and the contact force $\mathcal{R}^{\alpha}$ are respectively connected with $u$ and $r^{\alpha}$ through (6) and (8).

Here is the simplest example of such a law.

\subsection{FRICTIONLESS CONTACT}

In the notations of Subsec. 4.5, the assumption of no friction at the contact with label $\alpha$ consists in asserting that the contact force $\mathcal{R}^{\alpha}$ is normal to the tangent plane. Assume in addition that contact exhibits no cohesion (gluing) effect, in other words $\exists \rho_{\alpha} \geq 0: \mathcal{R}^{\alpha}=\rho_{\alpha} \mathbf{n}^{\alpha}$. It has been agreed to extend the definition of $\mathbf{n}^{\alpha}$ to the cases where $g_{\alpha}$, the normal gap, takes nonzero values and to put $\mathcal{R}^{\alpha}=0$ if $g_{\alpha}>0$. Define

$$
\mathcal{K}_{\alpha}(t, q):=\left\{\begin{array}{l}
\left\{\mathcal{V} \in \mathbf{E}_{3} \mid \mathcal{V} \cdot \mathbf{n}^{\alpha} \geq 0\right\} \quad \text { if } \quad g_{\alpha}(t, q) \leq 0 \\
\mathbf{E}_{3} \text { otherwise. }
\end{array}\right.
$$

This is the set of the values of the local right-velocity of $\mathcal{B}$ relatively to $\mathcal{B}^{\prime}$ (the latter may be an external boundary) which are compatible with noninterpenetration. In the first line, $\mathcal{K}_{\alpha}$ equals a half-space, so the normal cone $N_{\mathcal{K}_{\alpha}}$, evaluated at the origin $\mathbf{0}$ of $\mathbf{E}_{3}$, equals the half-line generated by $-\mathbf{n}^{\alpha}$. In case $\mathcal{K}_{\alpha}=\mathbf{E}_{3}$, the cone $N_{\mathcal{K}_{\alpha}}(\mathbf{0})$ reduces to the set $\{\mathbf{0}\}$.

Therefore, at time $t$, the no-friction and no-gluing assumptions, including the event of no-contact, are equivalent to assert

$$
-\mathcal{R}^{\alpha} \in N_{\mathcal{K}_{\alpha}(t, q)}(\mathbf{0}) .
$$

Let us consider a locally absolutely continuous motion $t \mapsto q(t)$ in the course of which the non-interpenetration condition $g_{\alpha}(t, q(t)) \geq 0$ remains satisfied, with a contact force verifying (11) for almost every $t$. Then the following is verified for almost every $t$

$$
-\mathcal{R}^{\alpha} \in N_{\mathcal{K}_{\alpha}(t, q)}\left(\mathcal{U}_{\alpha}\right) .
$$

In fact, this trivially holds when $g_{\alpha}(t, q(t))>0$, in view of the definition of $\mathcal{K}_{\alpha}$. At $t$ such that $g_{\alpha}(t, q(t))=0$, the right- and left-derivatives of the function $\tau \mapsto g_{\alpha}(\tau, q(\tau))$, if they exist, respectively are $\geq 0$ and $\leq 0$, so that 
the (bilateral) derivative, namely $\mathcal{U}_{\alpha} \cdot \mathbf{n}^{\alpha}$, which exists for almost every $t$, is zero. In this case, $\mathcal{K}_{\alpha}$ is a half-space and $\mathcal{U}_{\alpha}$ lies on its boundary, making that $N_{\mathcal{K}_{\alpha}(t, q)}\left(\mathcal{U}_{\alpha}\right)=N_{\mathcal{K}_{\alpha}(t, q)}(\mathbf{0})$.

Conversely, (12) implies (11) since the normal cone to $\mathcal{K}_{\alpha}$ at any point of $\mathbf{E}_{3}$ is a subset of $N_{\mathcal{K}_{\alpha}(t, q)}(\mathbf{0})$.

Inclusion (12) is a stronger requirement than (11). In particular, it compels $N_{\mathcal{K}_{\alpha}(t, q)}\left(\mathcal{U}_{\alpha}\right)$ to be nonempty, i.e. $\mathcal{U}_{\alpha} \in \mathcal{K}_{\alpha}(t, q(t))$. In view of the definition of $\mathcal{K}_{\alpha}$ and of the identity $\mathcal{U}_{\alpha} \cdot \mathbf{n}^{\alpha}=\partial g_{\alpha} / \partial t+\left\langle q^{\prime}, \nabla g_{\alpha}\right\rangle$, this reduces to the situation analyzed in Subsec. 2.2. By making $f=-g_{\alpha}$, one obtains:

Assume that (12) holds for almost every $t$ and that the non-interpenetration inequality $g_{\alpha} \geq 0$ holds at the initial instant. Then this inequality holds at every further instant.

This generally will be true whenever the contact with label $\alpha$ is governed by a contact law involving, among other stipulations

$$
\text { in all cases } \quad \mathcal{U}_{\alpha} \in \mathcal{K}_{\alpha} \text {. }
$$

If, in addition, this law secures the implication

$$
\mathcal{U}_{\alpha} \in \text { interior } \mathcal{K}_{\alpha} \Rightarrow \mathcal{R}^{\alpha}=0,
$$

as precisely (12) does, we call it a complete contact law.

Properties (13) and (14) are of importance in the construction of timestepping algorithms and also in the treatment of possible collisions [10][11]. Concerning the formulation of the Coulomb law of dry friction in the form of a complete contact law, see [11][5].

\subsection{ELIMINATION OF FRICTIONLESS CONTACT FORCES}

The following relationship is found to hold [11] between the element $\nabla f_{\alpha}$ of $\mathcal{Q}_{q}^{*}$ and the normal unit vector $\mathbf{n}^{\alpha}$ at point $M_{\alpha}$ to the contacting bodies, directed toward $\mathcal{B}$

$$
\exists \lambda_{\alpha} \geq 0 \text { such that } G_{\alpha}^{*} \mathbf{n}^{\alpha}=-\lambda_{\alpha} \nabla f_{\alpha} .
$$

The proof of this rests on a 'unilateral' version of the algebraic theorem of Lagrange multipliers, known in Convex Analysis as Farkas' lemma.

In the sequel, we shall assume that the mapping $G_{\alpha}$ is surjective of $\mathcal{Q}_{q}^{\prime}$ onto $\mathbf{E}_{3}$; equivalently, its transpose $G_{\alpha}^{*}$ is injective of $\mathbf{E}_{3}$ into $\mathcal{Q}_{q}^{* *}$. Then $\lambda_{\alpha}$ in (15) is nonzero. Some special positions of certain linkages may give rise to 'wedging' effects which contradict this assumption.

This allows one to apply Equ. (8), in order to eliminate the contact forces between the equation of Dynamics (9) and the law of frictionless 
contact (11), so obtaining the differential inclusion

$$
F(t, q, u)-A(t, q) \frac{d u}{d t} \in N_{\Phi(t)}(q) .
$$

The normal cone $N_{\Phi(t)}$ to the feasible region at time $t$ has been defined in Subseq. 4.4.

If, instead of (11), one invokes the complete law (12), the elimination of the contact forces rests on the definition of

$$
W(t, q):=\left\{v \in \mathcal{Q}_{q}^{\prime} \mid \forall \alpha \in J(t, q): \frac{\partial f_{\alpha}}{\partial t}+\left\langle v, \nabla f_{\alpha}\right\rangle \leq 0\right\},
$$

namely the set of the values of the right-derivative $q^{\prime+}(t)$ compatible with $q$ remaining in $\Phi(t)$. This is a closed convex polyhedral subset of $\mathcal{Q}_{q}^{\prime}$ whose normal cone at any point consists of a subset of $\mathcal{Q}_{q}^{*}$. One then obtains the differential inclusion

$$
F(t, q, u)-A(t, q) \frac{d u}{d t} \in N_{W(t, q)}(u) .
$$

Provided the non-interpenetration conditions (5) are satisfied at the intial instant, this differential inclusion, assumed to hold for almost every $t$, implies through the Integration Lemma that these conditions remain satisfied.

\subsection{TIME-STEPPING APPROXIMATION}

Let some initial conditions $q\left(t_{0}\right)=q_{0} \in \Phi\left(t_{0}\right)$ and $u\left(t_{0}\right)=u_{0} \in W\left(t_{0}, q_{0}\right)$ be imposed on a solution to the differential inclusion (18). Denote by $\left[t_{\mathrm{i}}, t_{\mathrm{f}}\right]$ a time-step, with length $h$. From the computed values $u_{\mathrm{i}}$ and $q_{\mathrm{i}}$ of the functions $u$ and $q$ at $t=t_{\mathrm{i}}$, which result from the preceding computation step, one has to predict $u_{\mathrm{f}}$ and $q_{\mathrm{f}}$ corresponding to $t_{\mathrm{f}}$.

The given functions $F$ and $A$ in (18) usually depend smoothly on their arguments, so we approximate their values throughout the concerned timestep by fixing $t$ as the 'midtime' $t_{\mathrm{m}}:=t_{\mathrm{i}}+h / 2$ and $q$ as the 'midposition' $q_{\mathrm{m}}:=q_{\mathrm{i}}+h u_{\mathrm{i}} / 2$ while $u$ is made equal to $u_{\mathrm{i}}$.

Similarly, the detection of the contacts to be treated as effective in the time-step is based on the values that the functions $f_{\alpha}$ take at point $\left(t_{\mathrm{m}}, q_{\mathrm{m}}\right)$, i.e. the set of the 'active' values of $\alpha$ is estimated to be $J_{\mathrm{m}}:=J\left(t_{\mathrm{m}}, q_{\mathrm{m}}\right)$ and $W(t, q)$ to equal $W_{\mathrm{m}}:=W\left(t_{\mathrm{m}}, q_{\mathrm{m}}\right)$.

We propose to evaluate the unknown $u_{\mathrm{f}}$ through a procedure of the implicit type. By using $\left(u_{\mathrm{f}}-u_{\mathrm{i}}\right) / h$ as an approximant of $d u / d t$, this yields

$$
h F\left(t_{\mathrm{m}}, q_{\mathrm{m}}, u_{\mathrm{i}}\right)-A\left(t_{\mathrm{m}}, q_{\mathrm{m}}\right)\left(u_{\mathrm{f}}-u_{\mathrm{i}}\right) \in N_{W_{\mathrm{m}}}\left(u_{\mathrm{f}}\right)
$$

(the right-hand side being a cone, the multiplier $h>0$ has been dropped). 
Therefrom $u_{\mathrm{f}}$ may be calculated as the solution of a Quadratic Programming problem. In fact there is no loss of generality by making $A$ equal the identity matrix, since this amounts to equip $\mathbf{R}^{n}$ with the 'kinetic' Euclidean metric. In view of a standard characterization of the proximal point of a point to the convex set $W_{\mathrm{m}}$, inclusion (19) is found equivalent to

$$
u_{\mathrm{f}}=\operatorname{prox}\left(W_{\mathrm{m}}, u_{\mathrm{i}}+h F_{\mathrm{m}}\right)
$$

Finally, one completes the computation step with $q_{\mathrm{f}}=q_{\mathrm{m}}+\frac{h}{2} u_{\mathrm{f}}$.

The above implicit time-stepping scheme provides a primitive example of the approach entitled 'Contact Dynamics'. Of course, more realistic contact laws than (12) may be introduced [11], in which case it proves efficient to avoid the elimination of the local variables $\mathcal{U}_{\alpha}, \mathcal{R}^{\alpha}$ and apply a nonlinear relaxation technique à la Gauss-Seidel [13]. This has been used in the simulation of Granular Materials involving thousands of bodies [12].

\section{References}

1. M. Anitescu, F. A. Potra and D. E. Stewart (1998) Time-stepping for threedimensional rigid body dynamics, in J. A. C. Martins and A. Klarbring (eds.), Computational Modeling of Contact and Friction, special issue of Computer Meth. in Appl. Mech. and Engng., to appear.

2. D. Baraff (1994) Fast contact force computation for non-penetrating rigid bodies, Computer Graphics (Proc. SIGGRAPH), 28, 23-34.

3. P. A. Cundall (1971) A computer model for simulating progressive large scale movements of blocky rock systems, Proceedings of the Symposium of the International Society of Rock Mechanics, Nancy, France, Vol.1, 132-150.

4. E. Delassus (1917) Mémoire sur la théorie des liaisons finies unilatérales, Ann. Sci. Ecole Norm. Sup., 34, 95-179.

5. G. de Saxcé and Z. Q. Feng (1991) New inequation and functional for contact with friction, J. Mech. of Struct. and Machines, 19, 301-325.

6. P. Lötstedt (1982) Mechanical systems of rigid bodies subject to unilateral constraints, SIAM J. Appl. Math., 42, 281-296.

7. M. D. P. Monteiro Marques (1993) Differential Inclusions in Nonsmooth Mechanical Problems: Shocks and Dry Friction, Birkhäuser, Basel, Boston, Berlin.

8. J. J. Moreau (1963) Les liaisons unilatérales et le principe de Gauss, Comptes Rendus Acad. Sci. Paris, 256, 871-874.

9. J. J. Moreau (1966) Quadratic programming in mechanics: dynamics of one-sided constraints, SIAM J. Control, 4, 153-158.

10. J. J. Moreau (1985) Standard inelastic shocks and the dynamics of unilateral constraints, in G. Del Piero and F. Maceri (eds.), Unilateral Problems in Structural Analysis, CISM Courses and Lectures, Vol. 288, Springer-Verlag, Wien, New York, 173-221.

11. J. J. Moreau (1988) Unilateral contact and dry friction in finite freedom dynamics, in J. J. Moreau and P. D. Panagiotopoulos (eds.), Nonsmooth Mechanics and Applications, CISM Courses and Lectures, Vol. 302, Springer-Verlag, Wien, New York, $1-82$.

12. J. J. Moreau (1997) Numerical investigation of shear zones in granular materials, in P. Grassberger and D. Wolf (eds.) Proc. HLRZ-Workshop on Friction, Arching, Contact Dynamics, World Scientific, Singapore, 233-247. 
13. J. J. Moreau (1998) Numerical aspects of the sweeping process, in J. A. C. Martins and A. Klarbring (eds.), Computational Modeling of Contact and Friction, special issue of Computer Meth. in Appl. Mech. and Engng., to appear.

14. L. Paoli and M. Schatzman (1993) Mouvements à un nombre fini de degrés de liberté avec contraintes unilatérales: cas avec perte d'énergie, Math. Modelling and Num. Anal., 27, 673-717.

15. F. Pfeiffer and C. Glocker (1996) Multibody Dynamics with Unilateral Contacts, John Wiley and Sons, New York. 